OPEN ACCESS

Edited by:

Francesca Patrignani,

Università di Bologna, Italy

Reviewed by:

Mariaelena Di Biase,

Istituto di Scienze delle Produzioni

Alimentari (ISPA), Italy

Paola Mattarelli,

Università di Bologna, Italy

${ }^{*}$ Correspondence:

Hongfei Zhao

zhaohf820603@163.com

zhaohf518@163.com

Specialty section

This article was submitted to

Food Microbiology,

a section of the journal

Frontiers in Microbiology

Received: 09 January 2018

Accepted: 01 May 2018

Published: 18 May 2018

Citation:

Zhou $F$, Jiang $X$, Wang $T$, Zhang $B$ and Zhao $H$ (2018) Lycium barbarum

Polysaccharide (LBP): A Novel

Prebiotics Candidate

for Bifidobacterium and Lactobacillus.

Front. Microbiol. 9:1034.

doi: 10.3389/fmicb.2018.01034

\section{Lycium barbarum Polysaccharide (LBP): A Novel Prebiotics Candidate for Bifidobacterium and Lactobacillus}

\author{
Fang Zhou', Xiaoying Jiang', Tao Wang1,2, Bolin Zhang ${ }^{1,2}$ and Hongfei Zhao ${ }^{1,2 *}$ \\ 'Department of Food Science, College of Biological Science and Biotechnology, Beijing Forestry University, Beijing, China, \\ ${ }^{2}$ Beijing Key Laboratory of Forest Food Processing and Safety, Beijing Forestry University, Beijing, China
}

Lycium barbarum is a boxthorn that produces the goji berries. The aim of the current study was to evaluate the proliferative effect of $L$. barbarum polysaccharides (LBP) on probiotics. LBP was extracted from goji berries and its monosaccharide composition characterized by gas chromatography (GC). The LBP extract contained arabinose, rhamnose, xylose, mannose, galactose, and glucose. LBP obviously promoted the proliferation of lactic acid bacteria ( $\mathrm{AB}$ ) strains, especially Bifidobacterium longum subsp. infantis Bi-26 and Lactobacillus acidophilus NCFM. In the presence of LBP in the growth medium, the $\beta$-galactosidase ( $\beta$-GAL) and lactate dehydrogenase $(\mathrm{LDH})$ activities of strain Bi-26 significantly increased. The activities of $\beta-G A L, L D H$, hexokinase $(H K)$, 6-phosphofructokinase (PFK), and pyruvate kinase (PK) of strain NCFM significantly increased under those conditions. LAB transcriptome sequencing analysis was performed to elucidate the mechanism responsible for the proliferative effect of LBP. The data revealed that LBP promoted the bacterial biosynthetic and metabolic processes, gene expression, transcription, and transmembrane transport. Pyruvate metabolism, carbon metabolism, phosphotransferase system (PTS), and glycolysis/gluconeogenesis genes were overexpressed. Furthermore, LBP improved cell vitality during freeze-drying and tolerance of the gastrointestinal environment. In summary, LBP can be used as a potential prebiotic for Bifidobacterium and Lactobacillus.

Keywords: Lycium barbarum polysaccharides, proliferation, transcriptome, stress conditions, activity

\section{INTRODUCTION}

Lactic acid bacteria (LAB) are important probiotics. Their beneficial effect on the host health has been widely recognized (Steinkraus, 2002). Because of their metabolic activities and fermentation products, LAB exert many physiological functions, e.g., immunostimulation, improved digestion and absorption, vitamin synthesis, inhibition of the growth of potential pathogens, cholesterol reduction, and reduction of gas distension (Wallace et al., 2011; Pandey et al., 2015). Therefore, 
LAB are widely used in the food industry (Corbo et al., 2014). Before LAB reach and colonize the human large intestine to fulfill their physiological functions, they must retain their viability during the following three stages: (i) storage; (ii) industrial processing, such as freeze-drying; and (iii) transit through the stomach and the small intestine (Figueroa-Gonzalez et al., 2011).

Prebiotics are usually non-digestible substances that exert positive effects on probiotic proliferation and host health. They are mainly oligosaccharides, polysaccharides, protein hydrolyzates, short-chain fatty acids, and plant and herb extracts (Wang et al., 2015). Supplementation of the growth medium with prebiotics is an effective way of promoting the proliferation of $\mathrm{LAB}$ and improving their viability under challenging conditions.

LAB strains can use various carbohydrates, a type of prebiotic, to produce energy required for their proliferation. Further, carbohydrates contain many hydroxyl groups; hence, they can form hydrogen bonds with polar groups on proteins to ensure protein stability, which could increase cell survival. Most prebiotics are short-chain carbohydrates with a degree of polymerization of two or more, e.g., inulin, fructooligosaccharides, and galactooligosaccharides (FigueroaGonzalez et al., 2011). Several studies have shown that some plant polysaccharides could be used as prebiotics, e.g., ginseng polysaccharides, Mangifera pajang fibrous polysaccharides, mushroom polysaccharides, and coconut polysaccharides (AlSheraji et al., 2012; Chou et al., 2013; He et al., 2015; Mohd Nor et al., 2017).

Lycium barbarum is a boxthorn that produces the goji berries. Goji berry is a traditional Chinese food material that can be used as a foodstuff and medicine. Polysaccharides are the most abundant group of active ingredients of the goji berry; they are composed of several types of monosaccharides (Kulczyński and Gramza-Michałowska, 2016). Currently, L. barbarum polysaccharides (LBP) are only infrequently used as prebiotics. Thus, the objectives of the current study were to: (i) characterize the monosaccharide composition of LBP; (ii) evaluate the effect of LBP on LAB proliferation; (iii) explore the mechanism of the proliferation by transcriptome analysis; and (iv) evaluate the protective effect of LBP on LAB under challenging conditions.

\section{MATERIALS AND METHODS}

\section{Preparation of LBP}

Dry fruits of $L$. barbarum were provided by Ningxia Senmiao Technology Group Co., Ltd. (Yin Chuan, China). Overall, 2 kg goji berries were used in the current study. Polysaccharides were extracted as described by Skenderidis et al. (2017) with some modification. Briefly, dry fruits were softened by soaking in distilled water $(1: 10, w / v)$ and then broken by using a juice extractor (Jiuyang, Shandong, China). The pulp was extracted with distilled water $(1: 20)$ for $3 \mathrm{~h}$ at $80^{\circ} \mathrm{C}$, centrifuged $(2000 \times g$ for $10 \mathrm{~min}$ ) to remove the fruit residue, and the supernatant vacuum-concentrated at $80^{\circ} \mathrm{C}$. The concentrate was then mixed with ethanol (95\%), and stored at $4^{\circ} \mathrm{C}$ for $12 \mathrm{~h}$. Finally, the precipitates were collected and freeze-dried, for a crude LBP preparation. The polysaccharides were purified (pigment and protein removed) by passing through a macroporous resin S-8 (Jinkai, Shanghai, China), as described by Yang et al. (2012).

\section{Gas Chromatography (GC) Analysis of LBP}

Glucose, arabinose, rhamnose, mannose, galactose, and xylose standards were provided by the National Standard Substances Center (Beijing, China). The polysaccharide content was determined by the anthrone-sulfuric acid method, using glucose as a standard (Dubois et al., 1956; Ren et al., 2013).

The monosaccharide composition of LBP was analyzed by GC. The LBP sample $(5 \mathrm{mg}$ ) was hydrolyzed in $4 \mathrm{~mL}$ of trifluoroacetic acid $(4 \mathrm{M})$ at $110^{\circ} \mathrm{C}$ for $3 \mathrm{~h}$. The trifluoroacetic acid was removed by evaporation with anhydrous ethanol. The hydrolyzate was derivatized by incubation with pyridine $(1.0 \mathrm{~mL})$, hexamethyldisilazane (HMDS; $0.6 \mathrm{~mL})$, and trimethylchlorosilane $\left(0.3 \mathrm{~mL}\right.$ ), at $50^{\circ} \mathrm{C}$ for $1 \mathrm{~h}$ (Song et al., 2011; Li et al., 2014). The samples were analyzed by GC (GC2010, Shimadzu, Japan) using a Shimadzu capillary column OV-1701 $(30 \mathrm{~m} \times 0.25 \mathrm{~mm} \times 0.33 \mu \mathrm{m})$. The analytical conditions were as follows: $0-5 \mathrm{~min}$ at $180^{\circ} \mathrm{C}$; ramp up $180^{\circ} \mathrm{C}$ to $230^{\circ} \mathrm{C}$ at $10^{\circ} \mathrm{C} / \mathrm{min}$; and hold for $20 \mathrm{~min}$ at $230^{\circ} \mathrm{C}$. The monosaccharides were quantified using an external standard method.

\section{Bacterial Strains and Culture Condition}

The LAB strains used in the current study were five Bifidobacterium strains: B. bifidum Bb-02, B. animalis subsp. lactis $\mathrm{Bi}-04, B$. longum subsp. infantis Bi-26, B. longum subsp. longum A6, and B. animalis BY-02; and two Lactobacillus strains: L. acidophilus NCFM and L. plantarum LP39. L. plantarum LP39 was obtained from the China Center of Industrial Culture Collection (CICC), while the other strains were provided by DuPont Co., Ltd. (Shanghai, China). Lactobacillus strains were cultured on de Man, Rogosa, and Sharpe (MRS) medium, while the Bifidobacterium strains were cultured anaerobically in MRS medium containing 0.05\% L-cysteine (Sigma-Aldrich, St. Louis, MO, United States). All strains were incubated at $37^{\circ} \mathrm{C}$ for $24 \mathrm{~h}$. The cells were collected by centrifugation $\left(4^{\circ} \mathrm{C}\right.$, $6000 \times g, 5 \mathrm{~min}$ ), washed twice with sterile saline solution, and resuspended in an equal volume of sterile saline before inoculation.

\section{Effect of LBP on the Proliferation of LAB Strains}

The effect of LBP on the proliferation of LAB strains was evaluated in three media. These were as follows: (i) control check (CK) medium, unsupplemented MRS medium; (ii) G1 medium, MRS medium supplemented with $0.5 \%$ LBP; and (iii) G2 medium, MRS medium containing 5\% LBP instead of 5\% glucose. Cell viability was confirmed by plate-counting on MRS agar after $37^{\circ} \mathrm{C}$ for $48 \mathrm{~h}$. For Bifidobacterium strains, L-cysteine $(0.05 \%)$ was added to the medium. All experiments were carried out in triplicate. 


\section{Effect of LBP on the Activities of Key Glucose Metabolism Enzymes}

The activities of key glucose metabolism enzymes were determined as follows. For Bifidobacterium strains, $\beta$-galactosidase ( $\beta$-GAL) and lactate dehydrogenase (LDH) activities were determined. For Lactobacillus strains, the activities of $\beta$-GAL, LDH, hexokinase (HK), pyruvate kinase $(\mathrm{PK})$, and 6-phosphofructokinase (PFK) were determined. Proteins were extracted by using the One Step Bacterial Active Protein Extraction kit (Sangon Biotech, Shanghai, China) and quantified using BCA Protein Assay kit (Sangon Biotech). The activities of key enzymes were determined using the appropriate enzyme activity kits according to the manufacturer's protocols (Suzhou Comin Biotechnology, Jiangsu, China). The enzyme activity was expressed as units (U) of activity per mg protein (U/mg prot).

\section{Transcriptome Sequencing}

In order to investigate the activation mechanism resulting from the presence of LBP, cells from G1 medium were analyzed and cells cultured in CK medium were used as control. Total RNA was extracted using the Trizol reagent (Invitrogen, Carlsbad, CA, United States). The rRNA was removed from RNA samples using the mRNA-only kit (Epicentre Biotechnologies, WI, United States). RNA concentration and purity were determined using Agilent 2100 Bioanalyzer (CA, United States). The mRNA was isolated from total RNA using Sera-Mag Magnetic oligo(dT) particles, and then chemically fragmented. The sequence library was constructed according to instructions for the ScriptSeq ${ }^{\mathrm{TM}}$ mRNA-Seq Library Preparation kit (Illuminacompatible). Samples were sequenced simultaneously using a single flow cell of the Illumina Hiseq2000 (CA, United States).

Quality reads were assembled into contigs, transcripts, and unigenes using the Velvet and Oases software. The reads per kilobase of exon model per million mapped reads value (RPKM) was used to normalize transcript abundances. A twofold differential was used to identify differentially expressed genes. All unigenes were used as queries in searching the $\mathrm{Nr}$ and SwissProt databases, and functionally annotated by GO analysis using the Blast2GO software ${ }^{1}$. Metabolic pathways were predicted by KEGG mapping.

\section{Effect of LBP on LAB Strains Exposed to Stress Conditions}

To explore the protective effect of LBP on LAB strains in stress conditions, freeze-drying process and gastrointestinal tolerance were performed in this study. The experiments were performed as specified below, with the activities of key enzymes used as the evaluation standard.

\section{Freeze-Drying Process}

Bacteria were incubated in the $\mathrm{CK}$ and $\mathrm{G} 1$ media at $37^{\circ} \mathrm{C}$ for $8 \mathrm{~h}$. The cells were collected by centrifugation $\left(4^{\circ} \mathrm{C}, 6000 \times g, 5 \mathrm{~min}\right)$ and mixed with lyophilized protective medium (sterile $12 \%$ skim

${ }^{1}$ https://www.blast2go.com milk). After freezing at $-20^{\circ} \mathrm{C}$ for $10 \mathrm{~h}$, samples were freezedried at $-55^{\circ} \mathrm{C}$ for $24 \mathrm{~h}$. The freeze-drying products were then immediately suspended in sterile saline, and the activities of key glucose metabolism enzymes determined.

\section{Simulated Gastrointestinal Conditions}

After incubation in the MRS medium for $8 \mathrm{~h}$, the cells were collected by centrifugation $\left(4^{\circ} \mathrm{C}, 6000 \times \mathrm{g}, 5 \mathrm{~min}\right)$, washed twice with sterile saline, and suspended in sterile saline at $10^{10} \mathrm{CFU} / \mathrm{mL}$. The suspensions were used in intestinal stress and gastric stress experiments (10\% inoculum). The simulated gastrointestinal conditions were created as described by Zhou et al. (2015) and Succi et al. (2017), with some modifications. To simulate the gastric juice, pepsin was added (Sigma-Aldrich, MO, United States) to the CK and G1 media for a final concentration of $10 \mathrm{U} / \mathrm{mL}$; the $\mathrm{pH}$ was adjusted to 2.0 using $1 \mathrm{~mol} / \mathrm{L} \mathrm{HCl}$. After inoculation with bacterial suspensions, the mixtures were incubated at $37^{\circ} \mathrm{C}$. Aliquots were withdrawn after $0,1.5$, and $3 \mathrm{~h}$, and viable bacterial counts were determined on MRS agar plates. To simulate the intestinal juice, bovine bile salts (3\%; SigmaAldrich) and trypsin ( $5 \mathrm{U} / \mathrm{mL}$; Sigma-Aldrich) were added to the $\mathrm{CK}$ and G1 media, and the $\mathrm{pH}$ adjusted to 7.5 using $1 \mathrm{~mol} / \mathrm{L}$ $\mathrm{NaOH}$. After inoculation with bacterial suspensions, the mixtures were incubated at $37^{\circ} \mathrm{C}$. Aliquots were withdrawn after $0,1.5$, and $3 \mathrm{~h}$, and viable bacterial counts were determined on MRS agar plates.

\section{Statistics}

All experiments and analyses were performed in triplicate, and the data are presented as the mean values. Microsoft Office Excel 2010 and SPSS (17.0) software were used for data analysis. One-way ANOVA and independent-sample $t$-test were used for statistical analysis. The significance threshold was set at $P<0.05$.

\section{RESULTS}

\section{Monosaccharide Composition of LBP}

As determined by the GC analysis (Figure 1), LBP was composed of arabinose, rhamnose, xylose, mannose, galactose, and glucose, at molar ratios of 0.18:0.81:0.07:2.17:0.23:6.52. At least four unknown components with higher content were detected. The top three known monosaccharides were glucose (29.38\%), mannose (9.88\%), and rhamnose (3.34\%).

\section{Proliferative Effect of LBP on LAB Strains}

The growth profiles of Bifidobacterium strains and Lactobacillus strains in LBP-supplemented media are shown in Table 1. LBP improved the growth of all tested Bifidobacterium strains except for strain Bi-04. The viable counts of Bb-02, Bi-26, A6, and BY-02 strains in the G1 medium exceeded those on the CK medium after $4 \mathrm{~h}$ of growth. The growth rates of different Bifidobacterium strains in the G2 medium were different. The viable counts of B. longum subsp. longum A6 in the G2 medium were higher than those in the CK medium but lower than those in the G1 medium. Among the strains, the growth of B. longum subsp. infantis $\mathrm{Bi}-26$ in the $\mathrm{CK}$ medium 
continuously increased over the $0-16 \mathrm{~h}$ period. However, cell numbers in the G1 and G2 media reached a maximum after $8 \mathrm{~h}$, which indicated that LBP shortened the logarithmic growth phase and stable phase of growth of strain Bi-26. The data (Table 1) indicated that the G1 medium supported bacterial growth better than the G2 and CK media. Strain Bi-26 was chosen for further study, and henceforth cultured in the G1 medium.

The optimal medium for the proliferation of Lactobacillus strains was also the G1 medium. As shown in Table 1, the viable counts of strain NCFM in the G1 medium reached 10.89 $(\log \mathrm{CFU} / \mathrm{mL})$, which was significantly higher than those in the CK medium $(P<0.05)$. Similar data were observed for the LP39 strain. However, LBP exerted the most pronounced proliferative effect on strain NCFM, which was consequently used in subsequent experiments.

\section{Effect of LBP on the Activities of Key Glucose Metabolism Enzymes}

The activities of key glucose metabolism enzymes were determined for B. longum subsp. infantis Bi-26 and L. acidophilus NCFM (Figure 2). The activities of key enzymes in strain Bi-26 and L. acidophilus NCFM were enhanced in the G1 medium. For strain Bi-26, the activities of $\beta$-GAL and LDH were higher after $8 \mathrm{~h}$ than after $12 \mathrm{~h}$ in this medium; for L. acidophilus NCFM, the activity of $\mathrm{HK}$ was higher at $12 \mathrm{~h}$, but the activity of PFK, PK, $\beta$-GAL, and LDH was higher at $8 \mathrm{~h}$.

\section{Transcriptome Analysis Gene Ontology Annotation}

To classify the predicted functions of unigenes, the gene ontology approach was employed. The most enriched GO terms are shown in Figure 3. For strain NCFM (Figure 3A), the most enriched GO terms were the cellular metabolic process (GO:0044237), cellular biosynthetic process (GO:0044249), heterocycle biosynthetic process (GO:0018130), primary metabolic process (GO:0044238), cellular nitrogen compound metabolic process (GO:0034641), gene expression (GO:0010467), nucleic acid-templated transcription (GO:0097659), nucleosidetriphosphatase activity (GO:0017111), and phosphorylation (GO:0016310). For B. longum subsp. infantis Bi-26 (Figure 3B), the most enriched GO terms were the cellular biosynthetic process (GO:0044249), cellular metabolic process (GO:0044237), gene expression (GO:0010467), macromolecule biosynthetic process (GO:0009059), primary metabolic process (GO:0044238), heterocyclic compound binding (GO:1901363), intracellular part (GO:0044424), catalytic activity (GO:0003824), aromatic compound biosynthetic process (GO:0019438), proteolysis (GO:0006508), and transcription DNA-templated (GO:0006351).

\section{Metabolic Pathway Analysis}

As shown in Table 2, four pathways were overexpressed and two pathways were suppressed when strain NCFM was grown in the presence of LBP. The overexpressed pathways were

TABLE 1 | Growth of strains in different media.

\begin{tabular}{|c|c|c|c|c|c|c|}
\hline Strain & Group & \multicolumn{5}{|c|}{ Colony forming units ( $\log \mathrm{cfu} / \mathrm{mL}$ ) } \\
\hline & G1 & $7.10 \pm 0.03$ & $8.43 \pm 0.16^{*}$ & $8.96 \pm 0.05$ & $10.60 \pm 0.09$ & $10.45 \pm 0.07^{*}$ \\
\hline & G2 & $7.05 \pm 0.02$ & $7.87 \pm 0.17^{*}$ & $8.79 \pm 0.11$ & $10.54 \pm 0.04$ & $10.44 \pm 0.13^{*}$ \\
\hline Bi-04 & CK & $7.79 \pm 0.03$ & $9.45 \pm 0.11$ & $10.03 \pm 0.03$ & $11.15 \pm 0.13$ & $11.17 \pm 0.03$ \\
\hline \multirow[t]{3}{*}{$\mathrm{Bi}-26$} & CK & $7.43 \pm 0.06$ & $9.35 \pm 0.08$ & $10.11 \pm 0.01$ & $10.75 \pm 0.07$ & $10.95 \pm 0.02$ \\
\hline & G1 & $7.35 \pm 0.07$ & $9.66 \pm 0.02^{* \#}$ & $10.35 \pm 0.04^{* \#}$ & $11.17 \pm 0.04^{* \#}$ & $11.18 \pm 0.02^{* \#}$ \\
\hline & G2 & $7.51 \pm 0.09$ & $9.31 \pm 0.02$ & $10.08 \pm 0.01$ & $10.98 \pm 0.06^{*}$ & $10.98 \pm 0.01$ \\
\hline \multirow[t]{2}{*}{ A6 } & CK & $7.62 \pm 0.10$ & $8.52 \pm 0.22$ & $8.94 \pm 0.23$ & $11.71 \pm 0.03$ & $10.54 \pm 0.02$ \\
\hline & G1 & $7.56 \pm 0.06$ & $9.25 \pm 0.09^{* \#}$ & $10.11 \pm 0.03^{* \#}$ & $11.81 \pm 0.05$ & $11.03 \pm 0.07^{*}$ \\
\hline \multirow[t]{3}{*}{ NCFM } & CK & $7.83 \pm 0.05$ & $9.35 \pm 0.05$ & $10.40 \pm 0.01$ & $11.05 \pm 0.01$ & $11.21 \pm 0.01$ \\
\hline & G1 & $7.75 \pm 0.08$ & $9.35 \pm 0.10$ & $10.89 \pm 0.01^{* \#}$ & $11.25 \pm 0.01^{* \#}$ & $11.26 \pm 0.05$ \\
\hline & G2 & $7.78 \pm 0.04$ & $9.34 \pm 0.02$ & $10.61 \pm 0.04^{*}$ & $11.07 \pm 0.02$ & $10.92 \pm 0.04$ \\
\hline \multirow[t]{3}{*}{ LP39 } & CK & $7.91 \pm 0.08$ & $9.16 \pm 0.12$ & $10.80 \pm 0.01$ & $11.30 \pm 0.02$ & $11.28 \pm 0.02$ \\
\hline & G1 & $7.85 \pm 0.03$ & $9.22 \pm 0.04$ & $10.97 \pm 0.01^{* \#}$ & $11.52 \pm 0.03^{* \#}$ & $11.64 \pm 0.02^{* \#}$ \\
\hline & G2 & $7.86 \pm 0.05$ & $8.45 \pm 0.15$ & $10.24 \pm 0.04^{*}$ & $11.31 \pm 0.02$ & $10.85 \pm 0.03^{*}$ \\
\hline
\end{tabular}

All results were repeated three times and expressed as mean \pm standard deviation of mean. ${ }^{*} P<0.05$ G1 and G2 compared with CK, ${ }^{*} P<0.05$ G1 compared with G2. 


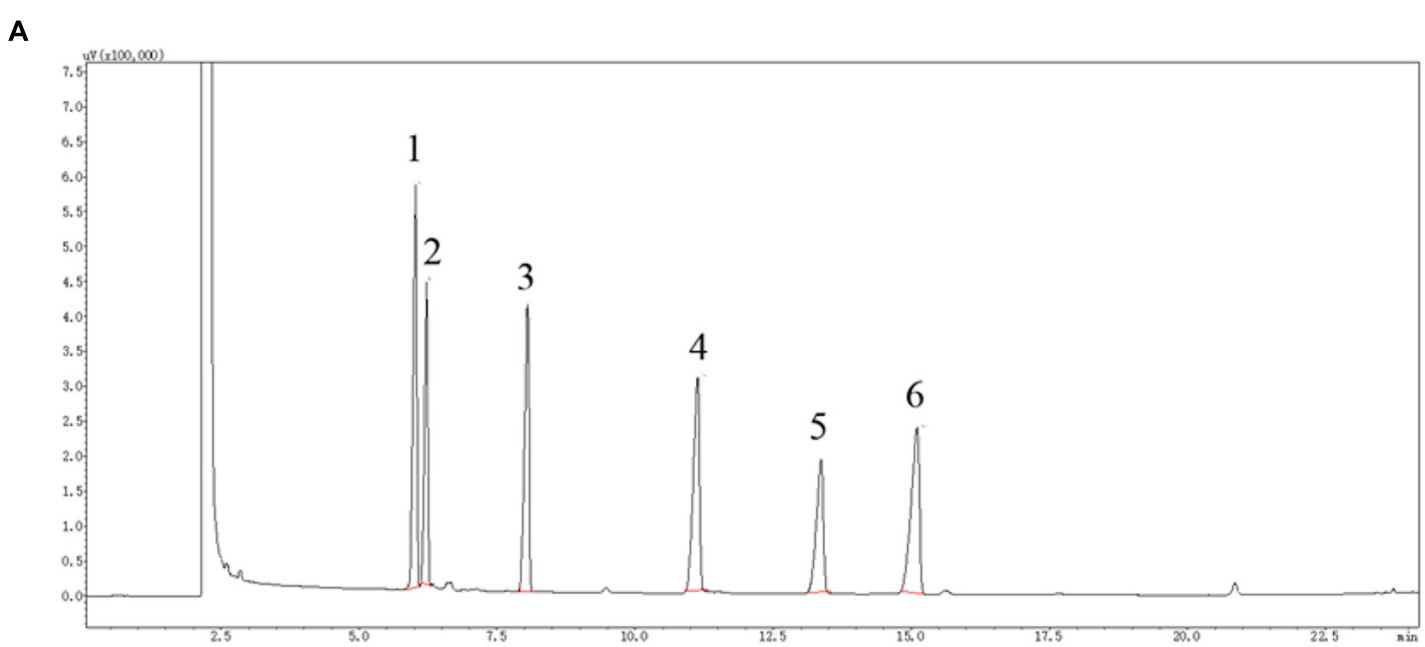

B

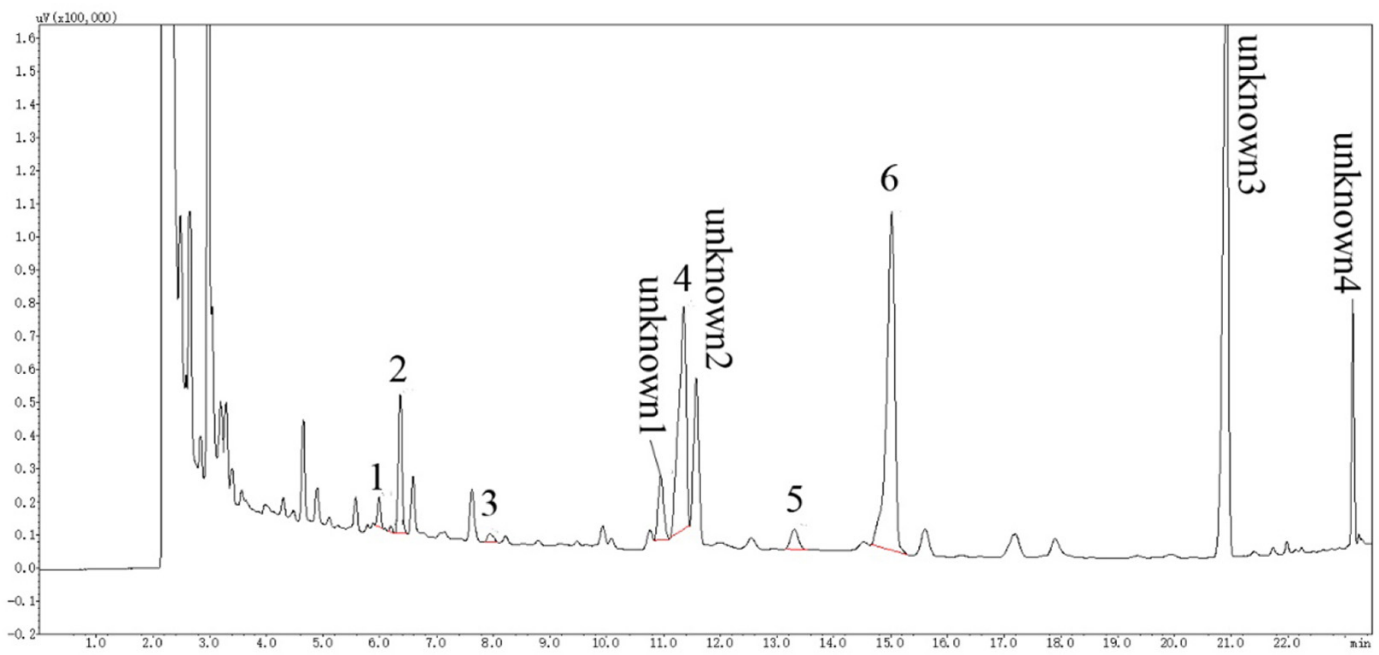

FIGURE 1 | GC chromatogram of of monosaccharides. (A) represents GC chromatogram of standard monosaccharides, according to the peak order: 1 Arabinose, 2 Rhamnose, 3 Xylose, 4 Mannose, 5 Galactose 6 Glucose; (B) is the GC chromatogram of LBP sample monosaccharides. The monosaccharide corresponding to the standard is labeled with the same number. In addition, at least four kinds of unknown components in LBP with higher concentration were detected.

pyruvate metabolism, carbon metabolism, phosphotransferase system (PTS), and glycolysis/gluconeogenesis. The pentose phosphate pathway and biosynthesis of amino acids were suppressed in this strain. In B. longum subsp. infantis Bi26 , the overexpressed genes were involved in the pathways of pyruvate metabolism, metabolic pathways, carbon metabolism, and biosynthesis of secondary metabolites. The suppressed pathways in this strain were involved in thiamine metabolism and microbial metabolism in diverse environments.

\section{Differential Expression Analysis}

As shown in Table 3, expression of seven genes was elevated in strain NCFM in the presence of LBP in the growth medium. These seven genes encoded phosphoenolpyruvate $(\mathrm{PEP})$ synthase ( $p p s)$, pyruvate formate-lyase-activating enzyme $(p f l A)$, formate C-acetyltransferase $(p f l B)$, nucleosidediphosphate kinase $(n d k)$, FAD-dependent glycerol-3-phosphate dehydrogenase $(g l p D)$, glycerol uptake facilitator protein $(g l p F 3)$, and glycerol uptake facilitator protein ( $g l p F 4)$. The expression of four genes was reduced under these conditions. They were genes encoding small heat shock protein (hsp1), translation initiation factor IF-3 (infC), transketolase ( $t k t 2)$, and cation (cobalt-zinc-cadmium) efflux protein $(c z c D 2)$.

In strain $\mathrm{Bi}-26$, five genes were overexpressed in the presence of LBP (Table 3). They encoded oligopeptide ABC transporter $(o p p A)$, dihydroxyacetone phosphotransferase (dak1A), copper 

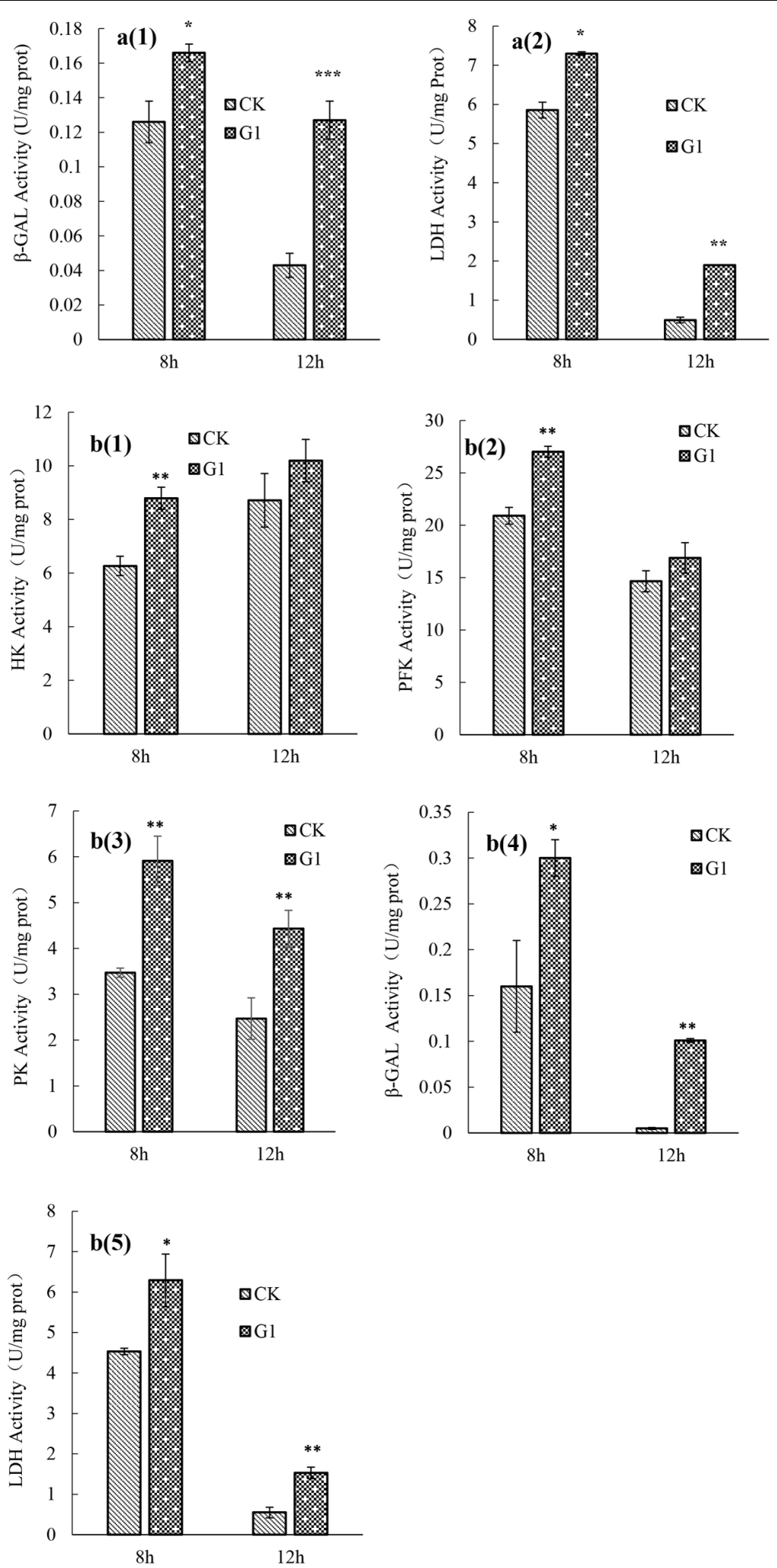

FIGURE 2 | key enzymes activity of strain Bi-26 and NCFM in control and LBP-supplemented MRS broth incubated at $37^{\circ} \mathrm{C}$ for 8 and 12 h. (a1) was $\beta$-GAL activity of Bi-26, (a2) was LDH activity of Bi-26. (b1) represent HK activity of NCFM, (b2) was PFK activity of NCFM, (b3) was PK activity of NCFM, (b4) was $\beta$-GAL activity of NCFM, (b5) represent LDH activity of NCFM. Error bars represent standard deviation ( $n \geq 3)$. *Means G1 significantly different from CK $P<0.05$; **Means G1 Significantly different from CK $P<0.01$; ***Means $\mathrm{G} 1$ significantly different from $\mathrm{CK} P<0.001$. 


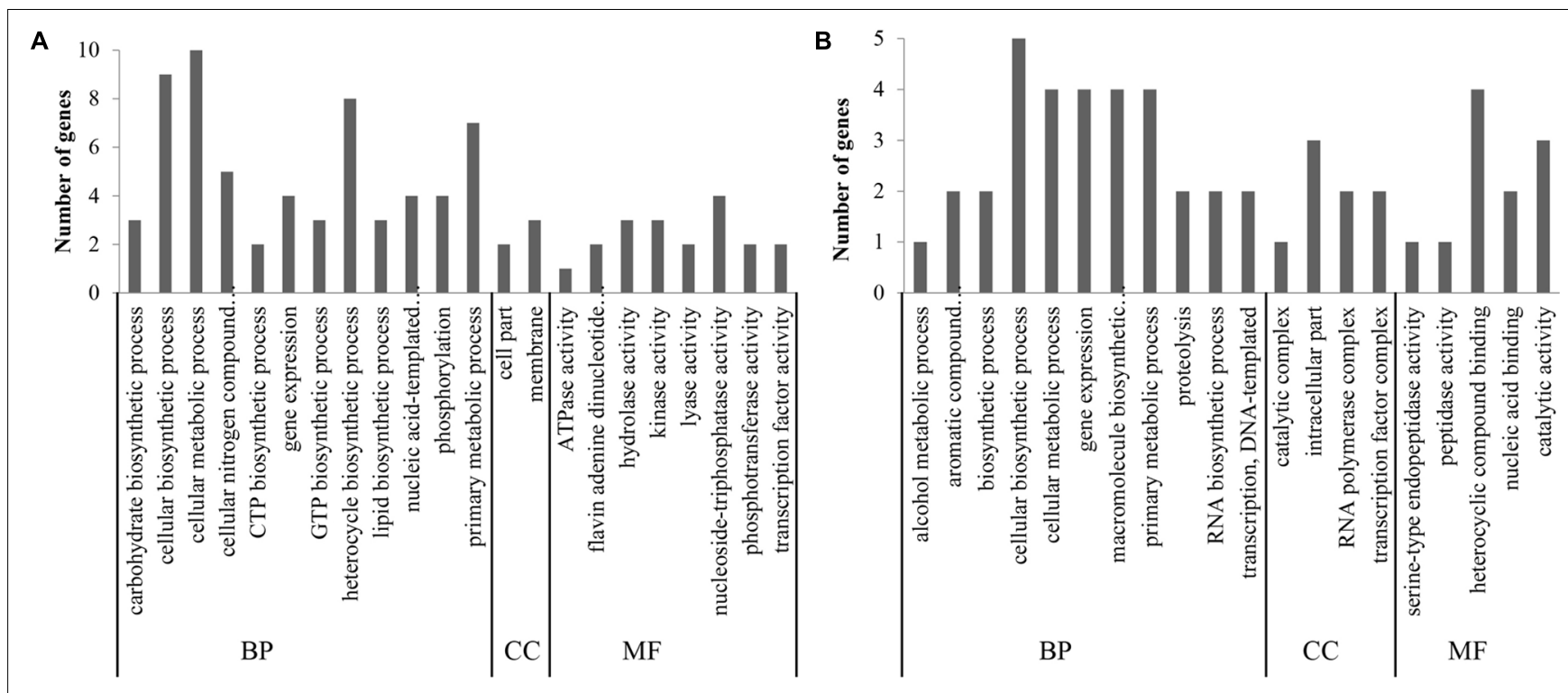

FIGURE 3 | The most enriched GO terms. (A) represents the result of strain NCFM. (B) was the data of strain Bi-26. The unigenes were classified into three main categories [biological process (BP), cellular component (CC) and molecular function (MF)].

TABLE 2 | Pathway analysis of genes.

L. acidophilus NCFM

Over-expressed

Pyruvate metabolism

Carbon metabolism

Phosphotransferase system (PTS)

Glycolysis / Gluconeogenesis
B. longum subsp. infantis $\mathrm{Bi}-26$

\begin{tabular}{ll} 
Over-expressed & Under-expressed \\
\hline Pyruvate metabolism & Thiamine metabolism \\
Metabolic pathways & Microbial metabolism in diverse environments \\
Carbon metabolism & \\
Biosynthesis of secondary metabolites &
\end{tabular}

TABLE 3 | Differential expression analysis.

\begin{tabular}{|c|c|c|c|c|}
\hline Strain & Over-expressed & log2. Fold change & Under-expressed & log2. Fold change \\
\hline & Pyruvate formate-lyase-activating enzyme (pflA) & 3.57 & Translation initiation factor IF-3 (infC) & 2.12 \\
\hline & Nucleoside-diphosphate kinase (ndk) & 2.31 & Cation (cobalt-zinc-cadmium) efflux protein (czcD2) & 2.87 \\
\hline & FAD-dependent glycerol-3-phosphate dehydrogenase (glpD) & 3.14 & & \\
\hline & Glycerol uptake facilitator protein (glpF3) & 2.65 & & \\
\hline \multirow[t]{5}{*}{$\mathrm{Bi}-26$} & Oligopeptide ABC transporter (oppA) & 2.56 & Transcription regulator of CopAB ATPases (copR) & 3.25 \\
\hline & Dihydroxyacetone phosphotransferase (dak1A) & 3.33 & Thiamin pyrophosphokinase (tpk) & 2.72 \\
\hline & Copper transporting ATPase (copA) & 14.88 & Xylulose-5-P phosphoketolase (xpkA) & 4.19 \\
\hline & Copper transporting ATPase (copB) & 12.79 & Ribulose-phosphate 3-epimerase (rpe) & 2.75 \\
\hline & Extracellular transglycosylase & 2.32 & & \\
\hline
\end{tabular}

transporting ATPase $(\operatorname{cop} A)$, copper transporting ATPase $(\operatorname{cop} B)$, and extracellular transglycosylase. The genes whose expression was reduced in this strain in the presence of LBP encoded transcription regulator of CopAB ATPases (copR), thiamin pyrophosphokinase $(t p k)$, xylulose-5-P phosphoketolase $(x p k A)$, and ribulose-phosphate 3-epimerase (rpe).

\section{The Protective Effect of LBP on LAB Strains Exposed to Stress Conditions} The Effect of LBP on Cell Survival After Freeze-Drying The activities of key enzymes of B. longum subsp. infantis Bi-26 and $L$. acidophilus NCFM after freeze-drying showed the same tendency in the presence of LBP (Table 4). Compared with cells 

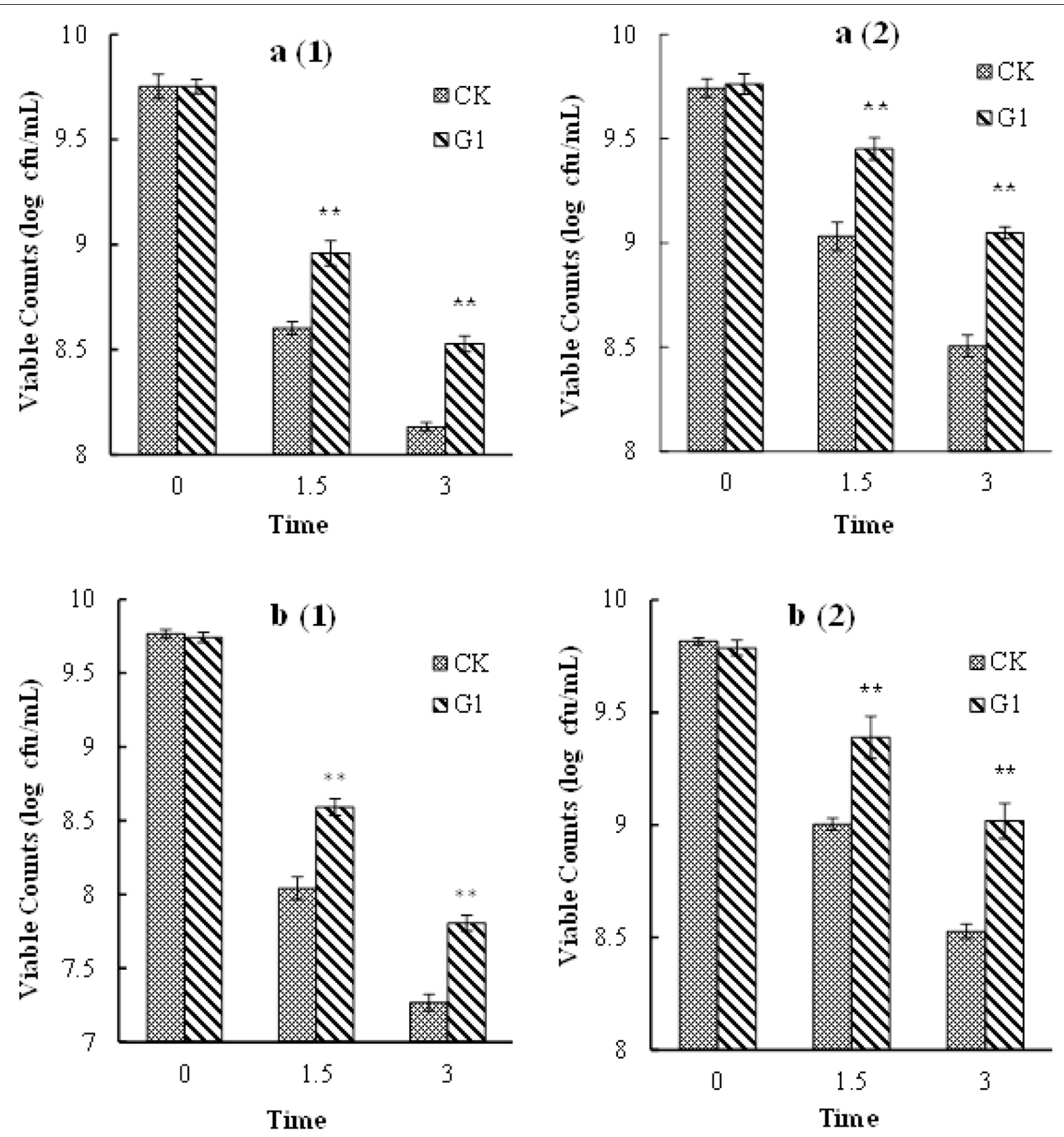

FIGURE 4 | Gastric and intestinal fluids tolerance of Bi-26 and NCFM. (a1) shows viable counts in gastric fluid of Bi-26, (a2) represents viable counts in intestinal fluid of Bi-26. (b1) was viable counts in gastric fluid of NCFM, (b2) was viable counts in intestinal fluid of NCFM. Error bars represent standard deviation ( $n \geq 3$ ). *Means G1 significantly different from CK at same time $P<0.05$;** Means G1 Significantly different from CK at the same time $P<0.01$.

grown in the CK medium (data not shown), the activities of key enzymes were significantly increased $(P<0.05)$ after growth in the G1 medium. This indicated that LBP improved the tolerance of strains Bi-26 and NCFM to freeze-drying by protecting the activity of key cellular enzymes.

\section{The Effect of LBP on Cell Gastric and Intestinal Tolerance}

The growth profiles of $B$. longum subsp. infantis Bi-26 and L. acidophilus NCFM in a simulated gastrointestinal environment are shown in Figure 4. The number of viable cells of strain Bi-26 and $L$. acidophilus NCFM was obviously reduced in the gastric fluid and intestinal fluid media. Bacterial tolerance of the gastric fluid medium was lower than that of the intestinal fluid medium. Compared with cells grown in the CK medium, the tolerance of the gastrointestinal environment of B. longum subsp. infantis Bi26 and L. acidophilus NCFM cells was improved by the presence of LBP; the number of viable cells was significantly higher than in the CK medium cells $(P<0.05)$ after $1.5 \mathrm{~h}$ and $3 \mathrm{~h}$ of stress exposure. 
TABLE 4 | The activities of key enzymes of strain Bi-26 and NCFM after freeze drying.

\begin{tabular}{|c|c|c|c|c|c|c|c|}
\hline \multirow[t]{2}{*}{ Group } & \multicolumn{2}{|c|}{ Bi-26 (U/mg prot) } & \multicolumn{5}{|c|}{ NCFM (U/mg prot) } \\
\hline & $\beta-G A L$ & LDH & HK & PFK & PK & $\beta-G A L$ & LDH \\
\hline CK & $0.184 \pm 0.006^{a}$ & $5.323 \pm 0.080^{c}$ & $7.260 \pm 0.082^{e}$ & $12.661 \pm 0.079^{9}$ & $4.803 \pm 0.008^{j}$ & $0.128 \pm 0.011^{m}$ & $2.330 \pm 0.075^{p}$ \\
\hline G1 & $0.200 \pm 0.008^{b}$ & $6.828 \pm 0.119^{d}$ & $9.807 \pm 0.065^{f}$ & $14.322 \pm 0.113^{h}$ & $5.451 \pm 0.012^{k}$ & $0.185 \pm 0.016^{n}$ & $4.886 \pm 0.094^{q}$ \\
\hline
\end{tabular}

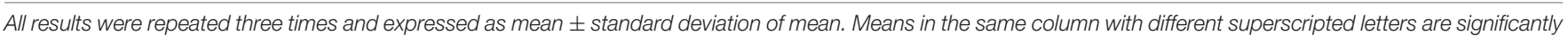
different $(P<0.05)$.

\section{DISCUSSION}

In the current study, the effect of LBP on the proliferation and survival of LAB was examined. The presented data suggested that the LAB strains differ with respect to their ability to use LBP. This was because the strains produce different types of glycosidases, whose activities were also different. In the current study, LBP promoted the proliferation of all tested $\mathrm{LAB}$ strains except strain $\mathrm{Bi}-04$. The growth of strain Bi04 was inhibited by LBP, which may have been associated with a high osmotic pressure exerted by LBP, leading to bacterial dehydration and growth inhibition (Wan et al., 2007). $B$. bifidum Bb-02, B. longum subsp. infantis Bi-26, B. longum A6, B. animalis BY-02, $L$. acidophilus NCFM, and $L$. plantarum LP39 were able to effectively utilize LBP. In summary, LBP can be used by various LAB because it is composed of different monosaccharides; consequently, it may be used as a prebiotic by many LAB.

Generally, LAB or strains of the species $L$. acidophilus produce lactic acid by EMP pathway. As is generally known, $\beta$-GAL, HK, PFK, PK, and LDH are the most important enzymes of carbohydrate metabolism (Cheng et al., 2014). Bifidobacterium strains produce lactic acid and acetic acid, etc., by a special HMP pathway. $\beta$-GAL and LDH play an important role in metabolic processes. Some other Bifidobacterium-specific enzymes of sugar metabolism, such as $\alpha$ - and $\beta$-glucosidase, mannosidase, and xylosidase were also in strain $\mathrm{Bi}-26$. In the current study, the activity of key glucose metabolism enzymes was enhanced by LBP. Carbohydrate metabolism significantly affects the growth of bacterial cell and the synthesis of secondary metabolites. The above-mentioned data indicated that LBP promotes the proliferation of $B$. longum subsp. infantis $\mathrm{Bi}-26$ and L. acidophilus NCFM.

In strain NCFM, genes overexpressed in the presence of LBP encoded several proteins involved in carbon metabolism (Pps, Pfla, PflB, GlpD, GlpF3, and GlpF4) and Ndk. Pps plays an essential role in glycolysis in the modified Embden-MeyerhofParnas pathway, converting pyruvate to PEP. PEP is a precursor in several biosynthetic processes (Farmer and Liao, 2001). PflA and PflB belong to the radical $S$-adenosylmethionine family of enzymes; they play important roles in one-carbon unit transfer (Crain and Broderick, 2014). GlpD, GlpF3, and GlpF4 are involved in glycerol metabolism. GlpD catalyzes the conversion of glycerol-3-phosphate to dihydroxyacetone phosphate. It is involved in the utilization of glycerol coupled to respiration. GlpF3 and GlpF4 are membrane proteins of the aquaporin family. Ndk is required for the synthesis of nucleoside triphosphates (NTP). It provides NTPs for nucleic acid synthesis, CTP for lipid synthesis, UTP for polysaccharide synthesis, and GTP for protein elongation and signal transduction. The data indicated that carbohydrate and energy metabolism were induced in the NCFM cells by LBP. This demonstrated that LBP promoted the proliferation of that strain. On the other hand, the expression of $h s p 1$, infC, $t k t 2$, and $c z c D 2$ genes was reduced in the presence of LBP (Table 3). Hsp1 is involved in the response to stress (e.g., temperature, hypertonicity, and amino acid deprivation). Its reduced gene expression indicated that LBP provided a uniform environment for bacterial cells. In other words, LBP enhanced the adaptability of cells to their microenvironment. Transketolase, encoded by $t k t 2$, catalyzes the reversible transfer of a two-carbon ketol unit from xylulose 5-phosphate to an aldose receptor. It plays an important role in the pentose phosphate pathway, which is a major source of the reducing power and metabolic intermediates for biosynthetic processes. Hence, reduced expression of the transketolase gene indicated that the cells absorbed various nutrients from the medium in the presence of LBP. Further, CzcD2 is thought to be an efflux pump that removes cobalt, zinc, and cadmium ions from the cell. These results suggested that LBP increased the anabolism and promoted cell proliferation.

In $B$. longum subsp. infantis $\mathrm{Bi}-26$, the following genes were overexpressed in the presence of LBP: opp, dak1A, $\operatorname{cop} A, \operatorname{cop} B$, and extracellular transglycosylase (Table 3). Transmembrane transport of oligopeptides requires the transport of a solute across a lipid bilayer. Opp is an ATP-dependent oligopeptide transporter and a member of the ATP-binding cassette (ABC) superfamily of transporters (Turroni et al., 2010). Since the gene oppA was overexpressed in Bi-26 cells in the presence of LBP (Table 3), this indicated that LBP promoted protein import. Dak1A converts dihydroxyacetone to the glycolytic intermediate dihydroxyacetone phosphate (Siebold et al., 2003). CopA is a protein with a copper-exporting ATPase activity, while CopB possesses a copper-importing ATPase activity. CopA and CopB can facilitate the transfer of a solute or solutes across the membrane. Extracellular transglycosylase plays an important role in the biosynthesis of peptidoglycan. Collectively, these data indicated that LBP enhanced the formation of the cell wall in strain Bi-26. Among the genes whose expression was reduced 
in Bi-26 cells in the presence of LBP were $\operatorname{cop} R$, tpk, and $x p k A$. CopR is a DNA-templated negative regulator of transcription. Phosphoketolases are key enzymes of the pentose phosphate pathway in heterofermentative and facultative homofermentative LAB. XpkA is a thiamine diphosphate (ThdP)-dependent enzyme found in Bifidobacterium sp. (Yin et al., 2005). The data therefore indicated that LBP stimulated the propagation of strain Bi-26 by enhancing the intracellular transport and synthesis.

Freeze-drying is one of the most effective methods for preserving microbial strain seeds. However, inevitably, cell viability is reduced during this process. As shown in the current study, LBP effectively improved the cell viability during freezedrying. Carvalho et al. (2004) reported that the survival of LAB strains during and after vacuum freeze-drying depended on the sugars present in the growth and drying media. Specific sugars, other than glucose, might underpin the distinct cell survival behavior during freeze-drying. As demonstrated in the current study, LBP mainly consists of arabinose, rhamnose, xylose, mannose, galactose, and glucose. Specific sugars might be produced by LAB strains during growth in a medium containing LBP that might exert a protective effect on LAB strains during freeze-drying. The protective effect may be also associated with the spatial distribution of the hydroxyl groups. The massive hydroxyl groups replace water molecules and maintain the spatial structure of bacterial proteins by interacting with the polar groups of protein via hydrogen bonds. This prevents protein denaturation associated with dehydration during freeze-drying (Carvalho et al., 2003; Yue et al., 2016).

To confer a health benefit on the host, the LAB must be able to overcome the physical and chemical barriers of the gastrointestinal tract, especially acid, proteolytic enzyme, and bile stresses (Nami et al., 2017). Consequently, LAB should be resistant to the gastrointestinal environment. As demonstrated in the current study, LBP improved the tolerance of B. longum subsp. infantis Bi-26 and L. acidophilus NCFM to the

\section{REFERENCES}

Al-Sheraji, S. H., Ismail, A., Manap, M. Y., Mustafa, S., and Yusof, R. M. (2012). Viability and activity of Bifidobacteria during refrigerated storage of yoghurt containing Mangifera pajang fibrous polysaccharides. J. Food Sci. 77, 625-630. doi: 10.1111/j.1750-3841.2012.02955.x

Blaiotta, G., Gatta, B. L., Capua, M. D., Luccia, A. D., Coppola, R., and Aponte, M. (2013). Effect of chestnut extract and chestnut fiber on viability of potential probiotic Lactobacillus strains under gastrointestinal tract conditions. Food Microbiol. 36, 161-169. doi: 10.1016/j.fm.2013.05.002

Carvalho, A. S., Silva, J., Ho, P., Teixeira, P., Malcata, F. X., and Gibbs, P. (2003). Effects of addition of sucrose and salt, and of starvation upon thermotolerance and survival during storage of freeze-dried Lactobacillus delbrueckii ssp. bulgaricus. J. Food Sci. 68, 2538-2541. doi: 10.1111/j.1365-2621.2003.tb07 057.x

Carvalho, A. S., Silvaa, J., Hob, P., Teixeiraa, P., Malcataa, F. X., and Gibbs, P. (2004). Relevant factors for the preparation of freeze-dried lactic acid bacteria. Int. Dairy J. 14, 835-847. doi: 10.1016/j.idairyj.2004.02.001

Cheng, X., Dong, Y., Su, P., and Xiao, X. (2014). Improvement of the fermentative activity of lactic acid bacteria starter culture by the addition of $\mathrm{Mn} 2+$. Appl. Biochem. Biotechnol. 174, 1752-1760. doi: 10.1007/s12010-014-1156-z gastrointestinal environment. In fact, numerous studies indicate that some prebiotics are able to exert a positive effect on the viability of LAB (Oliveira et al., 2009; Rattanaprasert et al., 2014; Succi et al., 2017). However, it remains unclear how prebiotics are able to affect the growth and resistance of LAB exposed to stress environments. From the previous reports (Even et al., 2002; Blaiotta et al., 2013), the possible mechanism was that the activities of key glucose metabolism enzymes were improved by LBP, it was helpful for fast recovery of bacteria. The hydrophobic effect of bile salts was also reduced by LBP. These effects were beneficial for $\mathrm{LAB}$, and enhanced their tolerance of the gastric fluid and intestinal fluid media.

\section{CONCLUSION}

LBP is a special carbon source, which contains arabinose, rhamnose, xylose, mannose, galactose, and glucose. It promotes the proliferation of some Bifidobacterium and Lactobacillus strains, possibly by enhancing the carbon and energy metabolism. It also exerts a protective effect on LAB strains exposed to stress conditions. Hence, LBP can be used as an industrial prebiotic candidate.

\section{AUTHOR CONTRIBUTIONS}

FZ conducted the experiments and wrote the manuscript. $\mathrm{XJ}$ and TW conducted the experiments. BZ provided some suggestions. $\mathrm{HZ}$ provided some ideas and checked the manuscript.

\section{FUNDING}

This work was supported by the National Natural Science Foundation of China (31601440) and the Fundamental Research Funds for the Central Universities (No. 2015ZCQ-SW-05).

Chou, W. T., Sheih, I., and Fang, T. J. (2013). The applications of polysaccharides from various mushroom wastes as prebiotics in different systems. J. Food Sci. 78, 1041-1048. doi: 10.1111/1750-3841.12160

Corbo, M. R., Bevilacqua, A., Petruzzi, L., Casanova, F. P., and Sinigaglia, M. (2014). Functional beverages: the emerging side of functional foods. Compr. Rev. Food Sci. Food Saf. 13, 1192-1205. doi: 10.1111/1541-4337. 12109

Crain, A. V., and Broderick, J. B. (2014). Pyruvate formate-lyase and its activation by pyruvate formate-lyase activating enzyme. J. Biol. Chem. 289, 5723-5729. doi: 10.1074/jbc.M113.496877

Dubois, M., Gilles, K. A., and Hamilton, J. K. (1956). Colorimetric method for determination of sugars and related substances. Anal. Chem. 28, 350-356. doi: 10.1021/ac60111a017

Even, S., Lindley, N. D., Loubière, P., and Cocaign-Bousquet, M. (2002). Dynamic response of catabolic pathways to autoacidification in Lactococcus lactis: transcript profiling and stability in relation to metabolic and energetic constraints. Mol. Microbiol. 45, 1143-1152. doi: 10.1046/j.1365-2958.2002. 03086.x

Farmer, W. R., and Liao, J. C. (2001). Precursor balancing for metabolic engineering of lycopene production in Escherichia coli. Biotechnol. Prog. 17, 57-61. doi: 10.1021/bp000137t 
Figueroa-Gonzalez, I., Quijano, G., Ramirez, G., and Cruz-Guerrero, A. (2011). Probiotics and prebiotics-perspectives and challenges. J. Sci. Food Agric. 91, 1341-1348. doi: 10.1002/jsfa.4367

He, Z., Wang, X., Li, G., Hao, Y., Zhang, J., Niu, C., et al. (2015). Antioxidant activity of prebiotic ginseng polysaccharides combined with potential probiotic Lactobacillus plantarum C88. Int. J. Food Sci. Tech. 50, 1673-1682. doi: 10.1111/ ijfs. 12824

Kulczyński, B., and Gramza-Michałowska, A. (2016). Goji Berry (Lycium barbarum): composition and health effects-a Review. Pol. J. Food Nutr. Sci. 66, 67-75. doi: 10.1515/pjfns-2015-0040

Li, Y., Yao, L., Wu, G., Pi, X., Gong, Y., Ye, R., et al. (2014). Antioxidant activities of novel small-molecule polysaccharide fractions purified from Portulaca oleracea L. Food Sci. Biotechnol. 23, 2045-2052. doi: 10.1007/s10068-0140278-y

Mohd Nor, N. N., Abbasiliasi, S., Marikkar, M. N., Ariff, A., Amid, M., Lamasudin, D. U., et al. (2017). Defatted coconut residue crude polysaccharides as potential prebiotics: study of their effects on proliferation and acidifying activity of probiotics in vitro. J. Food Sci. Technol. 54, 164-173. doi: 10.1007/s13197-0162448-9

Nami, Y., Haghshenas, B., and Khosroushahi, A. Y. (2017). Effect of psyllium and gum Arabic biopolymers on the survival rate and storage stability in yogurt of Enterococcus durans IW3 encapsulated in alginate. Food Sci. Nutr. 5, 554-563. doi: $10.1002 /$ fsn 3.430

Oliveira, R. P., Florence, A. C., Silva, R. C., Perego, P., Converti, A., Gioielli, L. A., et al. (2009). Effect of different prebiotics on the fermentation kinetics, probiotic survival and fatty acids profiles in nonfat symbiotic fermented milk. Int. J. Food Microbiol. 128, 467-472. doi: 10.1016/j.ijfoodmicro.2008.10.012

Pandey, K. R., Naik, S. R., and Vakil, B. V. (2015). Probiotics, prebiotics and synbiotics-a review. J. Food Sci. Technol. 52, 7577-7587. doi: 10.1007/s13197015-1921- 1

Rattanaprasert, M., Roos, S., Hutkins, R. W., and Walter, J. (2014). Quantitative evaluation of synbiotic strategies to improve persistence and metabolic activity of Lactobacillus reuteri DSM 17938 in the human gastrointestinal tract. J. Funct. Foods 10, 85-94. doi: 10.1016/j.jff.2014.05.017

Ren, A. N., Lu, Y., Zou, Y. F., and Shen, H. (2013). Separation, purification and preliminary structure analysis of acidic polysaccharides from safflower. Eur. Food Res. Technol. 237, 449-455. doi: 10.1007/s00217-013-2009-7

Siebold, C., Arnold, I., Garcia-Alles, L. F., Baumann, U., and Erni, B. (2003). Crystal structure of the Citrobacter freundii dihydroxyacetone kinase reveals an eight-stranded alpha-helical barrel ATP-binding domain. J. Biol. Chem. 278, 48236-48244. doi: 10.1074/jbc.M305942200

Skenderidis, P., Petrotos, K., Giavasis, I., Hadjichristodoulou, C., and Tsakalof, A. (2017). Optimization of ultrasound assisted extraction of Goji berry (Lycium barbarum) fruits and evaluation of extracts' bioactivity. J. Food Process Eng. 40:e12522. doi: 10.1111/jfpe.12522

Song, Y., Li, J., Hu, X., Ni, Y., and Li, Q. (2011). Structural characterization of a polysaccharide isolated from Lady Godiva Pumpkins (Cucurbita pepo lady godiva). Macromol. Res. 19, 1172-1178. doi: 10.1007/s13233-011-1102-7
Steinkraus, K. H. (2002). Fermentations in world food processing. Compr. Rev. Food Sci. Food Saf. 1, 23-32. doi: 10.1111/j.1541-4337.2002.tb00004.x

Succi, M., Tremonte, P., Pannella, G., Tipaldi, L., Cozzolino, A., Romaniello, R., et al. (2017). Pre-cultivation with selected prebiotics enhances the survival and the stress response of Lactobacillus rhamnosus strains in simulated gastrointestinal transit. Front. Microbiol 8:1067. doi: 10.3389/fmicb.2017.01067

Turroni, F., Bottacini, F., Foroni, E., Mulder, I., Kim, J. H., Zomer, A., et al. (2010). Genome analysis of Bifidobacterium bifidum PRL2010 reveals metabolic pathways for host-derived glycan foraging. Proc. Natl. Acad. Sci. U.S.A. 107, 19514-19519. doi: 10.1073/pnas.1011100107

Wallace, T. C., Guarner, F., Madsen, K., Cabana, M. D., Gibson, G., Hentges, E., et al. (2011). Human gut microbiota and its relationship to health and disease. Nutr. Rev. 69, 392-403. doi: 10.1111/j.1753-4887.2011.00402.x

Wan, R. F., Wang, L. P., and Jiang, S. X. (2007). Effects of two oligosaccharides on the multiplication of Lactobacilli in vitro. J. Clin. Rehabil. Tissue Eng. Res. 11, 3768-3770.

Wang, X., Huang, M., Yang, F., Sun, H., Zhou, X., Guo, Y., et al. (2015). Rapeseed polysaccharides as prebiotics on growth and acidifying activity of probiotics in vitro. Carbohydr. Polym. 125, 232-240. doi: 10.1016/j.carbpol.2015.02.040

Yang, R., Meng, D., Song, Y., Li, J., Zhang, Y., Hu, X., et al. (2012). Simultaneous decoloration and deproteinization of crude polysaccharide from pumpkin residues by cross-linked polystyrene macroporous resin. J. Agric. Food Chem. 60, 8450-8456. doi: 10.1021/jf3031315

Yin, X., Chambers, J. R., Barlow, K., Park, A. S., and Wheatcroft, R. (2005). The gene encoding xylulose-5-phosphate/fructose-6-phosphate phosphoketolase $(x f p)$ is conserved among Bifidobacterium species within a more variable region of the genome and both are useful for strain identification. FEMS Microbiol. Lett. 246, 251-257. doi: 10.1016/j.femsle.2005.04.013

Yue, L., Wang, J., Sa, R., Bao, Q., Tian, W., Chen, X., et al. (2016). Effects of culture conditions on the survival of freeze-dried lactic acid bacterial starter cultures. Food Sci. China 37, 270-276. doi: 10.1007/s00253-014-5592-z

Zhou, F., Zhao, H., Zheng, J., Dziugan, P., Li, S., and Zhang, B. (2015). Evaluation of probiotic properties of Lactobacillus strains isolated from traditional Chinese cheese. Ann. Microbiol. 65, 1419-1426. doi: 10.1007/s13213-014-0980-2

Conflict of Interest Statement: The authors declare that the research was conducted in the absence of any commercial or financial relationships that could be construed as a potential conflict of interest.

The reviewer PM and handling Editor declared their shared affiliation.

Copyright (c) 2018 Zhou, Jiang, Wang, Zhang and Zhao. This is an open-access article distributed under the terms of the Creative Commons Attribution License (CC BY). The use, distribution or reproduction in other forums is permitted, provided the original author(s) and the copyright owner are credited and that the original publication in this journal is cited, in accordance with accepted academic practice. No use, distribution or reproduction is permitted which does not comply with these terms. 